Pacific Journal of Mathematics

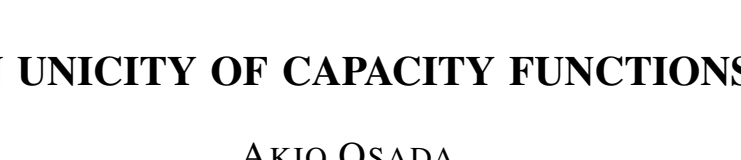




\title{
ON UNICITY OF CAPACITY FUNCTIONS
}

\author{
AkIo OSADA
}

\section{Sario's capacity function of a closed subset $\gamma$ of the ideal} boundary is known to be unique if $\gamma$ is of positive capacity. The present paper will determine the number of capacity functions of $\gamma$ in terms of the Heins harmonic dimension when $\gamma$ has zero capacity, under the assumption that $\gamma$ is isolated. This includes the special case where $\gamma$ is the ideal boundary.

1. Capacity functions. Denote by $\beta$ the ideal boundary of an open Riemann surface $R$ in the sense of Kerékjártó-Stoïlow. We consider a fixed nonempty closed subset $\gamma \subset \beta$ which is isolated from $\delta=\beta-\gamma$. Throughout this paper $D$ will denote a fixed parametric disk about a fixed point $\zeta \in R$ with a fixed local parameter $z$ and the uniqueness is always referred to this fixed triple $(\zeta, D, z)$. Here we do not exclude the case where $\gamma=\beta$.

For a regular region $\Omega \supset \bar{D}$ we denote by $\gamma_{\Omega}$ the part of $\partial \Omega$ which is "homologous" to $\gamma$. The remainder $\delta_{\Omega}=\partial \Omega-\gamma_{\Omega}$ consists of a finite number of analytic Jordan curves $\delta_{\Omega j}$. For a regular exhaustion $\left\{R_{n}\right\}_{n=0}^{\infty}$ with $R_{0} \supset \bar{D}$ and nonempty $\gamma_{R_{0}}$, set $\gamma_{n}=\gamma_{R_{n}}$ and $\delta_{n j}=\delta_{R_{n} j}$. Then there exists a unique function $p_{\gamma_{n}} \in H\left(R_{n}-\zeta\right)$ satisfying

(a) $p_{\gamma_{n}}|D=\log | z-\zeta \mid+h_{n}(z)$ with $h_{n} \in H(\bar{D})$ and $h_{n}(\zeta)=0$,

(b) $p_{\gamma_{n}} \mid \gamma_{n}=k_{n}(\gamma)$ (const.) and $p_{r_{n}} \mid \delta_{n j}=d_{n j}$ (const.) so that $\int_{\delta_{n j}} * d p_{\check{r}_{n}}=0$, which is called a capacity function of $\gamma_{n}$ (Sario [6]). It is known that $k_{n}(\gamma)$ increases with $n$ and the limit $k(\gamma)$ is independent of the choice of $\left\{R_{n}\right\}_{n=0}^{\infty}$. We call $e^{-k(\gamma)}$ the capacity of $\gamma$ and denote it by cap $\gamma$. When cap $\gamma>0, p_{\gamma_{n}}$ converges to a functions $p_{r}$, which is independent of the choice of the exhaustion (Sario [6]). Even when cap $\gamma=0$, we can also choose a subsequence of $\left\{p_{\gamma_{n}}\right\}$ which converges to a function $p_{r}$. Such functions $p_{r}$ will be called capacity functions of $\gamma$ (Sario [6]). As mentioned above there exists only one capacity function when cap $\gamma>0$.

It is the purpose of this paper to determine the number of capacity functions $p_{r}$ when cap $\gamma=0$.

2. The harmonic dimension of $\gamma$. Let $R, \beta, \gamma$ and $\delta$ be as in 1. Furthermore we suppose that $\gamma$ is of zero capacity. For a regular region $\Omega \supset \bar{D}$ we denote by $V_{Q i}$ components of $R-\bar{\Omega}$ whose derivations are contained in $\gamma$ and by $W_{\Omega j}$ the remaining components. Here an ideal boundary component will be called a derivation of $V_{\Omega i}$ when it is contained in the closure of $V_{\Omega i}$ in the compactification of $R$. Here- 
after we always choose $\Omega$ so large as to make the derivations of $W_{\Omega}=\bigcup_{j} W_{\Omega j}$ contain in $\delta$. Therefore $W_{\Omega}$ is always a neighborhood of all of $\delta$.

We consider the normal operator $L_{1}^{(2)}$ with respect to $R-\bar{\Omega}$ associated with the partition $P=\gamma+\sum_{j} \delta_{j}$ of $\beta$ where $\delta_{j}$ is a component of $\delta$ (Ahlfors-Sario [1]).

Let $q$ be a harmonic function in $R-\zeta$. Then $q$ will be called of $L_{1}$-type at $\delta$ when $q=L_{1}^{(2)} q$ in $W_{\Omega}$ for an admissible $\Omega$. It is easy to see that this property depends only on $\delta$, i.e., if $q=L_{1}^{(\Omega)} q$ in $W_{\Omega}$, then $q=L_{1}^{\left(\Omega^{\prime}\right)} q$ in $W_{\Omega^{\prime}}$ for every admissible $\Omega^{\prime}$.

We denote by $H P_{0}\left(V_{\Omega}\right)$ the family of functions $u$ such that $u$ is a positive harmonic function in $V_{\Omega}=\bigcup_{i} V_{\Omega i}$ with boundary values zero at $\gamma_{a}=\partial V_{a}$. We may extend $u$ to be identically zero in $W_{a}$. Moreover we consider the following two families of functions. The first family $N_{\Omega}$ consists of $u \in H P_{0}\left(V_{\Omega}\right)$ such that $\int_{r_{\Omega}} * d u=2 \pi$ where $\gamma_{\Omega}$ is positively oriented with respect to $\Omega$. The second family is the family $F$ of $q \in H(R-\zeta)$ having the following properties:

(c) $q|D=\log | z-\zeta \mid+h(z)$ with $h \in H(\bar{D})$ and $h(\zeta)=0$,

(d) $q$ is of $L_{1}$-type at $\delta$,

(e) $q$ is bounded from below near $\gamma$.

In addition to the obvious fact that $N_{\Omega}$ and $F$ are convex, they are related to each other as follows.

Lemma. There exists a bijective map $T$ of $N_{\Omega}$ onto $F$ satisfying (f) $T(\lambda u+(1-\lambda) v)=\lambda T u+(1-\lambda) T v$ for $u, v \in N_{\Omega}, 0<\lambda<1$, (g) $T u-u$ is bounded in $V_{\Omega}$.

For the proof let $u \in N_{\Omega}$ and denote by $L$ the direct sum of $L_{1}^{(\Omega)}$ and the Dirichlet operator with respect to $D$ (Sario [5]). Take the singularity function $s_{u}$ on $(R-\bar{\Omega}) \cup(D-\zeta)$ defined by $s_{u}=u$ in $R-\bar{\Omega}$ and $s_{u}=\log |z-\zeta|$ in $D-\zeta$. Since the total flux of $s_{u}$ is zero, the equation $p-s_{u}=L\left(p-s_{u}\right)$ has a unique solution $p_{u}$ on $R$, up to an additive constant. Normalize $p_{u}$ so as to satisfy (c) and set $T u=p_{u}$. Obviously $T u \in F$. Since $\gamma$ is of zero capacity, $T$ is clearly injective. The property in (f) and (g) follows easily from the definition of $T$.

To see the surjectivity let $q \in F$. We denote by $B q$ the bounded harmonic function in $V_{\Omega}$ with the boundary values $q \mid \gamma_{\Omega}$ at $\gamma_{\Omega}$. Set $u=q-B q$ in $V_{\Omega}$ and $u=0$ in $W_{\Omega}$. Since $q$ is of $L_{1}$-type at $o$ and bounded from below near $\gamma, u \in N_{\Omega}$. Therefore we have only to show that $q-s_{u}=L\left(q-s_{u}\right)$ in $(R-\bar{\Omega}) \cup(D-\zeta)$. By the definition of $u, q-u=B q$ in $V_{\Omega}$ and $L_{1}^{(\Omega)}(q-u)=L_{1}^{(\Omega)} q$ in $V_{\Omega}$. Furthermore $B q-L_{1}^{(\Omega)} q$ is bounded in $V_{\Omega}$ and vanishes on $\gamma_{\Omega}$. Hence $B q=L_{1}^{(\Omega)} q$ 
in $V_{\Omega}$. On the other hand, $L_{1}^{(\Omega)}(q-u)=L_{1}^{(\Omega)} q$ in $W_{\Omega}$. Consequently $q-u=L(q-u)$ also in $W_{\Omega}$. Finally it is obvious that the same equality holds in $D-\zeta$.

3. We denote by $M_{\Omega}$ the set of all minimal function in $H P_{0}\left(V_{\Omega}\right)$ normalized as $\int_{r_{\Omega}} * d u=2 \pi$. Lemma 2 guarantees that the cardinal number of $M_{\Omega}$ is independent of the choice $\Omega$. Extending Heins' definition (Heins [3]), we call it the harmonic dimension of $\gamma$, which we shall denote by $d_{r}$.

4. The number of capacity functions. We are now able to state our main result:

THEOREM. Suppose that $\gamma$ is an isolated closed subset of zero capacity in the ideal boundary of $R$. If the harmonic dimension of $\gamma$ is 1 , then the capacity function of $\gamma$ is unique. If the harmonic dimension of $\gamma$ is greater than 1 , there are a continuum of capacity functions of $\gamma$.

Denote by $C_{\gamma}$ the family of all capacity functions of $\gamma$, by $c_{\gamma}$ the cardinal number of $C_{r}$ and also by $\psi$ the cardinal number of the continuum. Then the statement of our theorem can also be summarized in a single formula as follows:

$$
c_{\gamma}=1+\left(d_{l}-1\right) \psi \text {. }
$$

5. Before entering the proof we need two lemmas, which will be used to show that $C_{\gamma}=F$. Let $R_{n}, \gamma_{n}$ and $\delta_{n j}$ be as in 1. Set $V_{n i}=V_{R_{n} i}$ and $W_{n j}=W_{R_{n} j}$ (see 2). Moreover put $\Omega_{0 n}=R-\bar{V}_{0}-\bar{W}_{n}$ with $V_{0}=\mathbf{U}_{i} V_{0 i}$ and $W_{n}=\mathbf{U}_{j} W_{n j}$.

Lemma. Let $p \in F$. Then there exists a sequence $\left\{p_{n}\right\}_{n=0}^{\infty}$ with $p_{n} \in H\left(\Omega_{0 n}-\zeta\right)$ satisfying

(h) $p_{n}|D=\log | z-\zeta \mid+h_{n}(z)$ with $h_{n} \in H(\bar{D})$ and $h_{n}(\zeta)=0$,

(i ) $p_{n} \mid \gamma_{0}=p+k_{n}$ (const.) and $p_{n} \mid \delta_{n j}=d_{n i}$ (const.) with

$$
\int_{\delta_{n j}} * d p_{n}=0,
$$

(j) $\left\{p_{n}\right\}$ converges uniformly to $p$ on any compact $K$ with

$$
\bar{K} \subset \Omega_{0}=R-\bar{V}_{0}-\zeta \text {. }
$$

For the proof construct $p_{n}$ with (h) and (i) by the linear operator method of Sario [5]. Denote by $D_{\varepsilon}$ a parametric disk about $\zeta$ with 
radius $\varepsilon$ and by $\alpha_{\varepsilon}$ its circumference. We orient $\alpha_{\varepsilon}$ and $\gamma_{0}$ negatively with respect to $\Omega_{0 n}-\bar{D}_{\varepsilon}$ and write according to Ahlfors-Sario [1]:

$$
A_{\varepsilon}(p)=\int_{\alpha_{\varepsilon}+\gamma_{0}} p^{*} d p, \quad B_{n}(p)=\int_{\hat{o}_{n}} p^{*} d p, \quad A_{\varepsilon}(p, q)=\int_{\alpha_{\varepsilon}+>0} p^{*} d q
$$

and

$$
B_{n}(p, q)=\int_{\delta n} p^{*} d q .
$$

For $m>n$ we denote by $D_{n, \varepsilon}\left(p_{m}-p_{n}\right)$ and $D_{n}\left(p_{m}-p_{n}\right)$ Dirichlet integrals of $p_{m}-p_{n}$ taken over $\Omega_{0 n}-\bar{D}_{\varepsilon}$ and $\Omega_{0 n}$ respectively. Since $B_{n}\left(p_{n}\right)=0, B_{n}\left(p_{n}, p_{m}\right)=0$,

$$
D_{n, \varepsilon}\left(p_{m}-p_{n}\right)=B_{n}\left(p_{m}\right)+2 A_{s}\left(p_{n}, p_{m}\right)-A_{s}\left(p_{n}\right)-A_{\varepsilon}\left(p_{m}\right) \text {. }
$$

Observing that $B_{n}\left(p_{m}\right)<0$ and letting $\varepsilon \rightarrow 0$,

$$
D_{n}\left(p_{m}-p_{n}\right) \leqq a_{m}-a_{n} \text { where } a_{j}=\int_{r_{0}} p^{*} d p_{j}+2 \pi k_{j} \quad(j=n, m) .
$$

Moreover we construct another sequence $q_{n} \in H\left(\Omega_{0 n}-\zeta\right)$ satisfying (h') $q_{n}|D=\log | z-\zeta \mid+h_{n}^{\prime}(z)$ with $h_{n}^{\prime} \in H(\bar{D})$ and $h_{n}^{\prime}(\zeta)=0$,

(i') $q_{n} \mid \gamma_{0}=p+k_{n}^{\prime}$ (const.) and the normal derivative of $q_{n}$ vanishes on $\delta_{n}$. By the same way as above we obtain

$$
D_{n}\left(q_{m}-q_{n}\right) \leqq b_{n}-b_{m} \text { where } b_{j}=\int_{\gamma_{0}} p^{*} d q_{j}+2 \pi k_{j}^{\prime} \quad(j=n, m)
$$

and

$$
D_{n}\left(p_{n}-q_{n}\right)=b_{n}-a_{n} .
$$

From (2), (3) and (4) we see $a_{n}$ is increasing and $b_{n}$ is decresing as $n$ increases and that $a_{n} \leqq b_{n}$. Therefore $\lim _{n} a_{n}$ and $\lim _{n} b_{n}$ exist and are finite. In particular it follows from (2) that $p_{n}$ converges uniformly to $p$ on any compact $K$ with $\bar{K} \subset \Omega_{0}$.

6. The following lemma is easy to see and plays an important role in the proof of our theorem.

Lemma. Let $p \in F$. Then there exist an exhaustion $\left\{R_{n}\right\}_{n=0}^{\infty}$ and a sequence $\left\{p_{n}\right\}_{n=0}^{\infty}$ with $p_{n} \in H\left(R_{n}-\zeta\right)$ having the properties (h) of Lemma 5 and

(k) $p_{n} \mid \gamma_{n}=p+k_{n}$ (const.) and $p_{n} \mid \delta_{n j}=d_{n j}$ (const.) with

$$
\int_{\delta_{n j}} * d p_{n}=0
$$

(1) $\left\{p_{n}\right\}$ converges uniformly to $p$ on any compact $K$ in $R-\zeta$. 
Since $\gamma$ has zero capacity we can see that there exists an Evans potential $e_{0}$ for $\gamma$, i.e., a function $e_{0} \in H(R-\zeta)$ satisfying the following conditions (Nakai [4]):

(m) $e_{0}|D=\log | z-\zeta \mid+w(z)$ with $w \in H(\bar{D})$ and $w(\zeta)=0$,

(n) $e_{0}$ is of $L_{1}$-type at $\delta$,

(o) $\lim _{z \rightarrow \gamma} e_{0}(z)=+\infty$.

Needless to say $e_{0} \in F$.

7. Proof of theorem. Consider $p_{\lambda}=\lambda e_{0}+(1-\lambda) q$ with a fixed $q \in F$ and $0<\lambda<1$. It is clear that $\lim _{z \rightarrow r} p_{\lambda}(z)=+\infty$ and $p_{\lambda} \in F$. Therefore by Lemma 6 we obtain

$$
\left\{p_{\lambda}\right\}_{0<i<1} \subset C_{\gamma} \text {. }
$$

On the other hand, obviously

$$
C_{\gamma} \subset F \text {. }
$$

Moreover observe that $\lambda \rightarrow p_{\lambda}$ is injective if $e_{0} \neq q$.

By the approximation theorem of Heins [2], we can see at once that if $d_{r}=1$, so is the cardinal number of $F$. It is trivial that the converse is valid. Hence $c_{r}=1$ if and only if $d_{r}=1$.

Suppose that $d_{r} \geqq 2$. Then there exists a $q \in F$ with $q \neq e_{0}$. By the injectivity of $\lambda \rightarrow p_{\lambda}, \psi \leqq c_{\gamma}$. Conversely it follows from (6) that $c_{\gamma} \leqq$ the cardinal number of $F$ which is not greater than $\psi$. Thus $c_{\gamma}=\psi$. In either case, since $d_{\gamma} \leqq \psi$, we have $c_{r}=1+\left(d_{r}-1\right) \psi$.

The author would like to express his warmest thanks to Professor Nakai for his kind guidance. He is also grateful for the valuable comments of the refree.

\section{REFERENCES}

1. L. V. Ahlfors and L. Sario, Riemann surfaces, Princeton Univ. Press, Princeton, N. J., 1960.

2. M. Heins, A lemma on positive harmonic functions, Ann. of Math. 52 (1950), 568-573.

3. _- Riemann surfaces of infinite genus, Ann. of Math. 55 (1952), 296-317.

4. M. Nakai, On Evans patential, Proc. Japan Acad. 38 (1962), 624-629.

5. L. Sario, A linear operator method on arbitrary Riemann surfaces, Trans. Amer. Math. Soc. 72 (1952), 281-295.

6. Capacity of the boundary and of a boundary component, Ann. of Math. 59 (1954), 135-144.

7. L. Sario and K. Noshiro, Value distribution theory, D. Van Nostrand, 1966.

Received October 2, 1967 and in revised form February 27, 1968. This is a part of the author's thesis for the partial satisfaction of the degree Master of Science at Nagoya University. 



\section{PACIFIC JOURNAL OF MATHEMATICS}

\section{EDITORS}

H. ROYDEN

Stanford University

Stanford, California

R. R Phelps

University of Washington

Seattle, Washington 98105
J. DUGUNDJI

Department of Mathematics

University of Southern California

Los Angeles, California 90007

RICHARD ARENS

University of California

Los Angeles, California 90024

\section{ASSOCIATE EDITORS}
E. F. BECKENBACH
B. H. NeumanN
F. WOLF
K. YosidA

\section{SUPPORTING INSTITUTIONS}

UNIVERSITY OF BRITISH COLUMBIA

CALIFORNIA INSTITUTE OF TECHNOLOGY

UNIVERSITY OF CALIFORNIA

MONTANA STATE UNIVERSITY

UNIVERSITY OF NEVADA

NEW MEXICO STATE UNIVERSITY

OREGON STATE UNIVERSITY

UNIVERSITY OF OREGON

OSAKA UNIVERSITY

UNIVERSITY OF SOUTHERN CALIFORNIA
STANFORD UNIVERSITY

UNIVERSITY OF TOKYO

UNIVERSITY OF UTAH

WASHINGTON STATE UNIVERSITY

UNIVERSITY OF WASHINGTON

AMERICAN MATHEMATICAL SOCIETY CHEVRON RESEARCH CORPORATION TRW SYSTEMS

NAVAL WEAPONS CENTER 


\section{Pacific Journal of Mathematics

Vol. 28, No. $1 \quad$ March, 1969

Patrick Robert Ahern, On the geometry of the unit ball in the space of real annihilating measures .............................. 1

Kirby Alan Baker, Equational classes of modular lattices ............. 9

E. F. Beckenbach and Gerald Andrew Hutchison, Meromorphic minimal surfaces ......................................... 17

Tae Ho Choe, Intrinsic topologies in a topological lattice ..............

John Bligh Conway, A theorem on sequential convergence of measures and

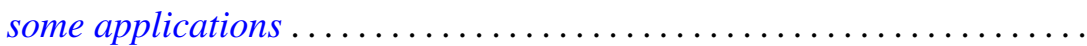

Roger Cuppens, On the decomposition of infinitely divisible probability laws without normal factor.

Lynn Harry Erbe, Nonoscillatory solutions of second order nonlinear

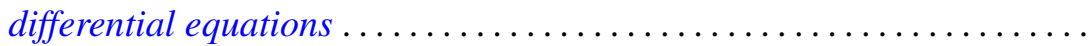

Burton I. Fein, The Schur index for projective representations of finite

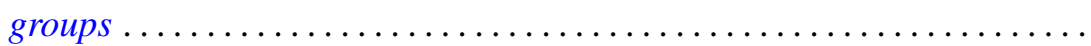

Stanley P. Gudder, A note on proposition observables............... 101

Kenneth Kapp, On Croisot's theory of decompositions ............... 105

Robert P. Kaufman, Gap series and an example to Malliavin's theorem . . . 117

E. J. McShane, Robert Breckenridge Warfield, Jr. and V. M. Warfield,

Invariant extensions of linear functionals, with applications to measures and stochastic processes ................

Marvin Victor Mielke, Rearrangement of spherical modifications ...

Akio Osada, On unicity of capacity functions ..............

Donald Steven Passman, Some 5/2 transitive permutation groups ...

Harold L. Peterson, Jr., Regular and irregular measures on groups and dyadic spaces...

Habib Salehi, On interpolation of $q$-variate stationary stochastic processes...

Michael Samuel Skaff, Vector valued Orlicz spaces generalized

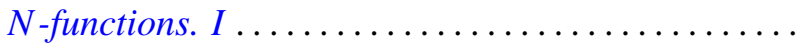

A. J. Ward, On $H$-equivalence of uniformities. II...........

Thomas Paul Whaley, Algebras satisfying the descending chain condition for subalgebras...

G. K. White, On subgroups of fixed index

Martin Michael Zuckerman, A unifying condition for implications among the axioms of choice for finite sets ................. 